\title{
Téoros
}

Revue de recherche en tourisme

\section{La Cité de l'Énergie : mettre en valeur le patrimoine industriel de Shawinigan}

\author{
Normand Cazelais, Benoît Gauthier et Jean-Marc Carpentier
}

Volume 15, numéro 2, été 1996

Patrimoine industriel

URI : https://id.erudit.org/iderudit/1075026ar

DOI : https://doi.org/10.7202/1075026ar

Aller au sommaire du numéro

Éditeur(s)

Université du Québec à Montréal

ISSN

0712-8657 (imprimé)

1923-2705 (numérique)

Découvrir la revue

Citer cet article

Cazelais, N., Gauthier, B. \& Carpentier, J.-M. (1996). La Cité de l’Énergie : mettre en valeur le patrimoine industriel de Shawinigan. Téoros, 15(2), 36-40.

https://doi.org/10.7202/1075026ar d'utilisation que vous pouvez consulter en ligne. 


\section{La Cité de l'Énergie:}

\section{METTRE EN VALEUR LE PATRIMOINE INDUSTRIEL DE SHAWINIGAN}

Normand Cazelais, journaliste et géographe. Hydro-Québec Benoît Gauthier, coordonnateur muséologie/muséographie La Cité de l’Énergie

Jean-Marc-Carpentier, responsable du concept muséologique et du contenu thématique de la Cité de l'Énergie

La Niagara de l'est. C'est ainsi que l'on désignait Shawinigan dans la première noitié du XXe siecle. Comme Niagara, Shawinigan possédait l'un des plus grands complexes hydroélectriques d'Amérique du Nord et réunissait sur son territoire de grandes industries énergivores et à la fine pointe de la technologie : aluminiun, électrochinie, pâtes et papiers.

Jusqu'à la Révolution tranquille, Shawinigan a été un puissant catalyseur de la croissance industrielle du Québec. Depuis cette époque, l'activité industrielle de la ville n'a cessé de décliner. Aujourd'hui, elle est à redéfinir ses assises économiques. Un des outils dont elle dispose pour relancer sa croissance est de puiser à mêne un patrimoine industriel riche et exemplaire pour développer son industrie touristique. C'est pour mettre en valeur ce patrimoine industriel et stimuler le tourisme au Centre-de-la-Mauricie que la Cité de l'Énergie a été créée.

\section{SHAWINIGAN, D'HIER À AUJOURD'HUI}

Le potentiel touristique de Shawinigan ne date pas d'hier.

Dans Le Devoir ${ }^{\mathrm{t}}$ du 8 juillet 1996 , le journaliste Jean Pichette souligne que, déjà en 1865 , le Times de Londres vantait les beautés des chutes du Saint-Maurice à cet endroit et que, quelques années plus tard, des peintres comme Comelius Krieghoff et Otto Reinhold Jacobi, membres du célèbre Groupe des Sept, ont peint ce paysage «sauvage - le Niagara de $l^{\prime}$ Est, disait-on - d'une qualité vraiment hors de l'ordinaire.

La révolution industrielle a aussi reconnu les possibilités du site. Première ville du
Canada à être dotée d'un plan d'urbanisme en 1899 , qualifiée de acité électriquew, créée ade rien* par la Shawinigan Water and Power, elle demeure avant tout, écrit Pichette, sun symbole de l'entrée du Québec dans l'ère industrielle $w^{2}$. Construit en 1947 à l'époque où s'y versaient les plus hauts salaires au Canada, l'hôtel de ville rappelle, par sa stature, cette prospérité d"antan: en 1904, fait unique au pays, Shawinigan regroupait près de ses chutes la première aluminerie canadienne (la Pittsburgh Reduction Co. qui deviendra l'Alcan), une usine de pâtes et papiers (la Belgo), une usine électrochimique (la Shawinigan Carbide) et surtout deux centrales hydroélectriques pour alimenter ces industries energivores.
A l'aube du XXe siècle, Shawinigan n'a jamais connu les horizons promis et sa gloire appartient davantage au passé, après de longues années de stagnation économique. Mais son histoire et sa géographie sont toujours vivantes: au coeur du foyer et du paysage industriels qu'elles ont suscités, les chutes de Shawinigan constituent encore l'elément porteur de la Cité de l'Énergie.

\section{LA PETITE HISTOIRE D'UN GRAND PROJET}

C"est dans un contexte de restructuration économique qu'a pris forme le projet de doter Shawinigan et sa région d'un attrait touristique, culturel, récréatif et éducatif d'envergure nationale et internationnale. Pour réaliser ce projet "grandiose", les promoteurs bénéficient d'un site naturel d'une beauté exceptionnelle - le site des chutes de Shawinigan - et de la présence sur celui-ci d'un patrimoine industriel d'une grande richesse, hautement représentatif non seulement de l'histoire de Shawinigan mais aussi d'une ctape capitale de l'industrialisation du Québec.

Ce projet a commencé à germer dans les années $1970^{3}$, au moment où s'est enclenché le processus de décroissance industrielle et qu'il devenait évident qu"une relance de l'économie par la grande industrie était chose du passé. Mais c'est au début des années 1980 que le projet a véritablement pris forme. $\AA$ cette époque, Hydro-Québec a initié plusieurs études et travaux de recherche qui ont conduit à la première mouture, en 1985, du projet de Centre d'interprétation 
de l'industrie de Shawinigan et à la constitution d'une Corporation sans but lucratif pour le mettre en oeuvre.

Le coût considérable des immobilisations et l'important déficit d'opération anticipé en retardent cependant la réalisation. Hydro-Québec hésite à s'engager seule dans l'affaire. En 1988, le projet est relancé avec l'entrée en scène de nouveaux partenaires : Alcan, ConsolidatedBathurst, Division Belgo, les Villes de Shawinigan et de Shawinigan-Sud. Des solutions sont apportées pour rentabiliser les operations et l'aide des deux gouvernements est sollicitée pour financer une partie des immobilisations. Le gouvernement du Québec reconnaît rapidement l'intérêt du projet, mais il exige qu'il soit révisé et actualisé avant de s'impliquer financièrement. En 1989,

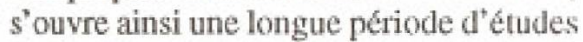
ponctuée de multiples rencontres de négociations avec les deux paliers de gouvernement. Quatre ans plus tard, toutes ces discussions, études, rencontres et reformulations du projet débouchent sur une proposition d'aide financière conjointe des deux gouvernements totalisant $9 \mathrm{M} \$$. Cette somme, à laquelle stajoute une mise de fonds de la Corporation, permettra de réaliser une partie importante des installations de l'île Melville, soit le Centre de sciences et la tour d'observation. Les équipements récréatifs de l'île Melville et le recyclage à des lins muséologiques des bâtiments patrimoniaux d'Alcan sont reportés à une étape ulturieure. L'année suivante, la proposition est officialisée. Pour sa part, HydroQuébec accorde une subvention de 9,15 M\$ afin de défrayer le coût des immobilisations projetées sur sa propriçté. Au total, le coût du projet s'élève à $19,7 \mathrm{M}$.

La Cité de l'Énergie a donc êtế créce dans le but de faire revivre au plus large public possible la remarquable aventure industrielle de Shawinigan et de contribuer au développement de l'industrie récréotouristique de la région. Elle s'inscril également sur la carte des sites du patrimoine industriel du Québec comme un témoin spectaculaire de l'industrialisátion du pays et comme un maillon impor= tant du réseau des musées de science et de technique.

\section{UN PROJET FONDÉ SUR LE PARTENARIAT}

La mise en oeuvre de la Cité de l'Énergie repose principalement sur la synergie qui stest développée entre les nombreux partenaires impliqués dans le projet. Leur implication n'est pas limitée seulement au financement du projet; ils participent activement à toutes les étapes de sa mise oeuvre. Outre le suivi administratif, ils agissent aussi comme conseillers de la Corporation pour baliser les orientations et fournir un soutien technique et professionnel à l'équipe de direction.

De manière plus concrete, cene collaboration des partenaires à la réalisation du projet s'exprime par la participation aux différents comités consultatifs mis sur pied par la Cité de l'Énergie. Ces comités sont au nombre de quatre. Le suivi général du projet est assuré par le comité de coordination qui rassemble des représentants des différents ministères fédérraux et provinciaux impliqués dans le financement du projet, de ses autres partenaires tinanciers, d'experts externes et de représentants de la Corporation. Ce comité recueille les avis des autres comités consultatifs et fait des recommandations au Conseil d'administration sur les décisions à prendre et les actions à mellie en oeuvre.

Trois autres comilés ont été constitués pour assurer la mise en oeuvre de ce projet. Il y a d'abord le comile scientifique. Son mandat consiste a conseiller la Cornoration sur les diverses facettes de la mission musénle de l'institution en cours d'implantation et sur' les orientations en matière de contenu thếnatique, de moyens muscographiques, d'animatlion et d'éducation. Ce comité demeurera en place après l'ouverture de l'établissenent au public pour agir à titre de comite conseil pour toutes questions se rapportant aux aspects muxứctux de l'institution.

Un comité de constunction a été mis sur pied pour la dune du projet. II foumit des avis sur les differents aspects de la consuruction, comme l'établissement de politiques d'acquisition des biens et services, l'élude des plins et devis, des soumissions, etc. Enlin, un connité de minkeling eflectue le suivi de ta méparattion du plan de maketing et de communication en cours de rílisilion.

\section{LA STRATÉGIE DE MISE EN VALEUR ET DE POSITIONNEMENT TOURISTIQUE}

La stratégie tant de mise en valeur que de positionnement touristiques de la Cité de l'Énergie s'articule jusqu'ici autour de quelques traits fondamentaux: originalité et unicité d'un produit à la fois récréatif et éducatif, thématique d'intétêt contemporain, éloignement relatif de grands centres de population, nécessité conséquente d'attirer un achalandage extérieur et d'intégrer le site dans la dynamique touristique de la Mauricie.

Le futur Centre d'interprétation table en effet sur le côté spectaculaire du site, la qualité du traitement thématique et la diversité de l'expérience proposée pour attirer des visiteurs. L'objectif est de l'inscrire parmi les grandes institutions muséologiques du Québec et d'en faire une force d'appel amajeures aux côtés d'autrés établissements québécois situés dans des aconditions d'isolement semblable qui réussissent à attirer chaque année plus de 100000 visiteursw. Au Québec, le Village de Val-Jalbert, la Pulperie de Chicoutimi, le Village québécois d'antan, le Jardin zoologique de Saint-Félicien et, ailleurs au Canada, Science Nord à Sudbury, Upper Canada Village à Morrisburg et le Village acadien à Caraquet sont autant d'exemples cités à titre de références.

Même si le Centre "colle d'abord et avant tout à l'histoire de Shawinigan, une ville industrielle dans le XXe siècles, ses promoteurs sont convaincus que son propos, qui s'attachera à l'histoire et à la technologie industrielles en général devrait

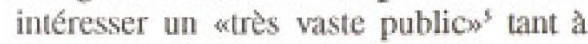
$l^{+}$échelle nationale qu' internationale:

* $\bar{A}$ cette fin, le contenu scientifique et technologique sera vulgarisé et organisé de manière à susciter l'intérêt aussi bien des personnes âgées que celui des jeunes adultes ou d'adolescents. Dans ce sens, (il) s'inscrit dans le courant de l'importance grandissante accordée à la culture scientifique au cours des dernieres annees, et cela dans tous les pays industrialises. Le visiteur percevra (...) les relations étroites entre le développement des sciences 


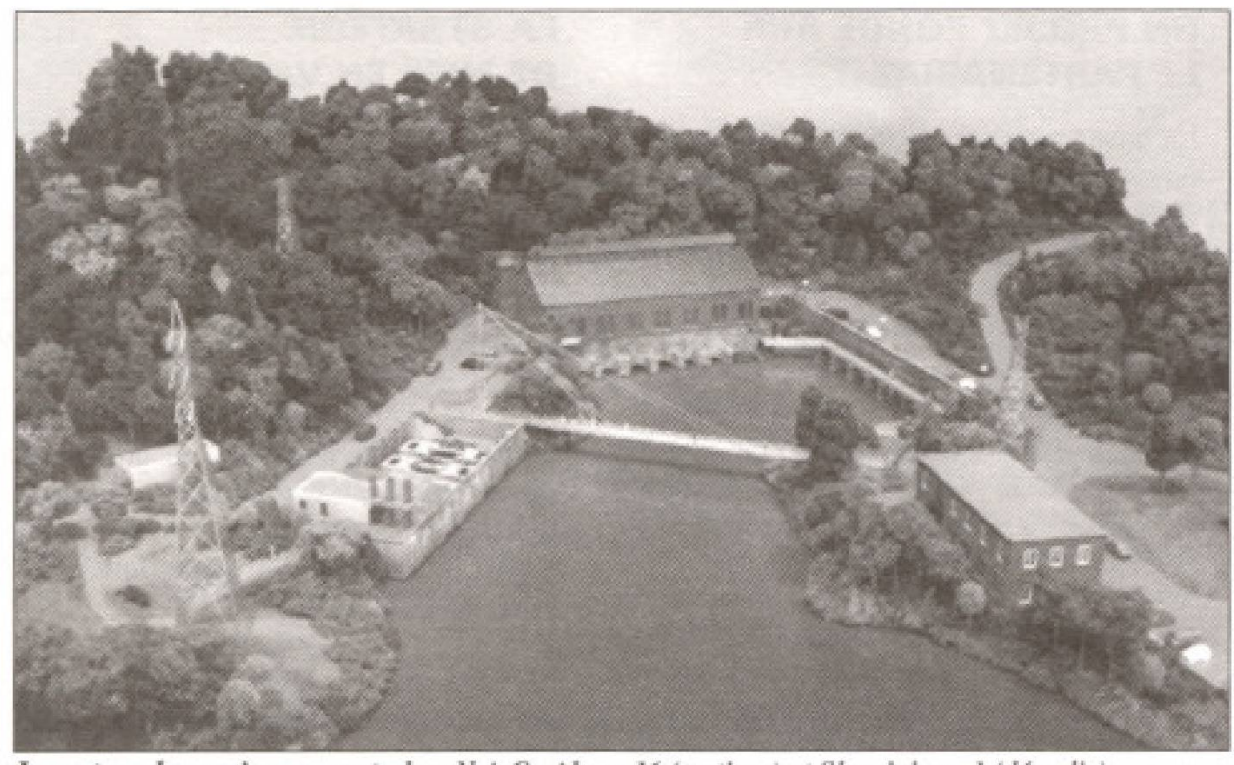

Le secteur des anciennes centrales: N.A.C., Alcan-I6 (vestiges) et Shawimigan I (dénalie).

et des technologies d'une part, et celui de l'économie et des sociêtés d"autre part. $x^{6}$

L'animation se modulera selon les saisons: plus discrète en été pour *assurer un contact humain, (...) diversifiée et adaptée aux clients selon les endroits ouverts le reste de l'année $w^{\top}$. Dans cet esprit, la clientèle scolaire constituera un important actif pour étoffer la fréquentation à l'automne, en hiver et au printemps.

Comme dans la plupart des établissements museaux ouverts toute l'année, le Centre entend connaître $70 \%$ de sorr achalandage, soit quelque 103000 visiteurs, au cours des treize semaines comprises entre la mi-juin et la mi-septembre. Ce phénomène, note le Programme de mise en oeuvre, est «plus marqué dans les établissements situés en région qui ont une clientèle scolaire plus petite et où le tourisme hivernal est axé sur des activités qui sont plutôt concurrentes (motoneige, ski) que complémentaires aux activités du Centre $\omega^{*}$.

La frequentation annuelle prévue se situe a la hauteur de 150000 visiteurs et devrait, espère-t-on, être atteinte après trois ans d'exploitation".

Le tableau ci-contre présente l'achalandage prévu lors des cinq premières années d'exploitation, qui s'appuie sur un taux de croissance annuelle uniforme de
$5 \%$ pour chaque catégorie de visiteurs. Notant qu'il est "peu probable que cette croissance se poursuive au mêtme rythme atu-delà de cette périodes, des études en marketing "établissent, pour la troisième année d'exploitation, la ventilation géographique comme suit: clientèle régionate $(36,9 \%)$, clientèle du Québec et hors région $(39,1 \%)$, clientèle hors Québec $(8,4 \%)$ et groupes spécialisés inntégrant les groupes scolaires et d'âge d'or de toute provenance $(15,6 \%)$.

Le Centre d'interprétation de l'industrie de Shawinigan veut se positionner comme un eproduit de consommation a spectre larges" ${ }^{14}$ centre muséographique, présentation multimédiạ, vestiges historiques, installations industrielles en exploitation, environnement naturel, vul- garisation scientifique et technologique, etc. Pour ajouter à ses propres forces, il entend profiter du potentiel touristique de la Mauricie en se posant comme un produit d'appel récréatif et éducatif, pour contribuer à la wformation d"une masse critique touristique dans la région ${ }^{12}$.

D'où la volonté manifeste de s'insérer au coeur du corridor touristique du SaintMaurice, "voie de passage de plus en plus fréquentée par les touristes québécois et étrangers se dirigeant vers le Lac-SaintJean (et qui) $s^{7}$ affirme de plus en plus comme une destination de vacances ${ }^{13}$ grâce aux activités de plein air qu'on peut y pratiquer (Parc national de la Mauricie, réserves fauniques, pourvoiries, zecs), à des événements touristiques populaires (Festival western de Saint-Tite, Classique internationale de canot) et à quelques pôles d'attraction (Forges du SaintMaurice, Village d'Émilie).

Pour sa part, Michel Charpentier, de I'Association touristique de Mauricie/Bois-Francs, woit d'un bon oeil la venue d'une telle citê de l'Énergie: «La région, souligne-t-il, enregistre beaucoup de voyages-personnes mais ces visiteurs y dépensent moins que dans d'autres régions du Québec. Avec le Parc de la Mauricie, le Centre peut devenir une deuxième locomotive apte a garder les gens plus longtemps et à permettre au taux de rétention touristique de la région, qui est de 2,6 jours présentement, de rejoindre celui du Québec à 3,1 jours. Et puis, avec l'Hôtel des Postes dans les Bois-Francs, le Musée du Bronze a Inverness, Le Baluchon à Saint-Paulin et le tout nouveau Musée des arts et traditions populaires à Trois. Rivières, la région commence à avoir un produit qui s'exporte bien.* ${ }^{44}$

\section{TABLEAU DE LA FRÉQUENTATION ANNUELLE (PRÉVISIONS)}

\begin{tabular}{l|l|l|l||l|l} 
CLIENTELES & AN 1 & AN 2 & AN 3 & AN 4 & AN 5 \\
\hline Residents & 48627 & 51420 & 51754 & 54342 & 57059 \\
\hline Villegiateurs & 2328 & 2444 & 2567 & 2695 & 2830 \\
Touristes & 61671 & 64755 & 67992 & 71392 & 74962 \\
\hline Groupes Age d'or & 4648 & 4880 & 5124 & 5381 & 5850 \\
Groupes scolaires & 17224 & 18085 & 18989 & 19939 & 20936 \\
\hline TOTAL & 134496 & 141584 & 146426 & 153749 & 161437 \\
\hline
\end{tabular}




\section{UN SITE AUX ATTRAITS MULTIPLES}

La Cité de l'Énergie, dont l'ouverture au public aura lieu en juin 1997, englote la majeure partie du site industriel des chutes de Shawinigan. Deux grandes zones sont viskes par ce projet. Tout d'abord, l'ìle Melville où seront érigés le Centre de sciences et la tour d'observation. Puis, le secteur Hydro-Québec qui comprend l'ensemble des installations qui forment le Complexe hydrólectrique de Shawinigan.

L'ĩe Melville est le coeur thématique de la Cité de l'Énergie. C'est là qué sont regroupés l'essentiel des services (stationnement, accueil, billetterie, restauration, boutique). Sur les quelque 2700 mètres carrés de cette nouvelle construction, la moitiế est consacrée à la muscographie. Ces espaces comprennent la salle multimédia, la salle d'exposition pemanente, la salle des. expositions temporaires et itinerantes, l'observatoire de la tour et une sille d'animation pédagogique. À tout cela s'ajoutent des locaux administratifs et techniques.

L'aceès au secteur Hydro-Québec se fait par' un bateau qui relie l'íle Melville au Complexe Shawinigan. La, les activités de la Citế de l'Énergie sont rếparties en quatre zones principales. Les déplacements entre ces différents points d'attraction se feront à bord d'un véhicule spécialement aménagé.

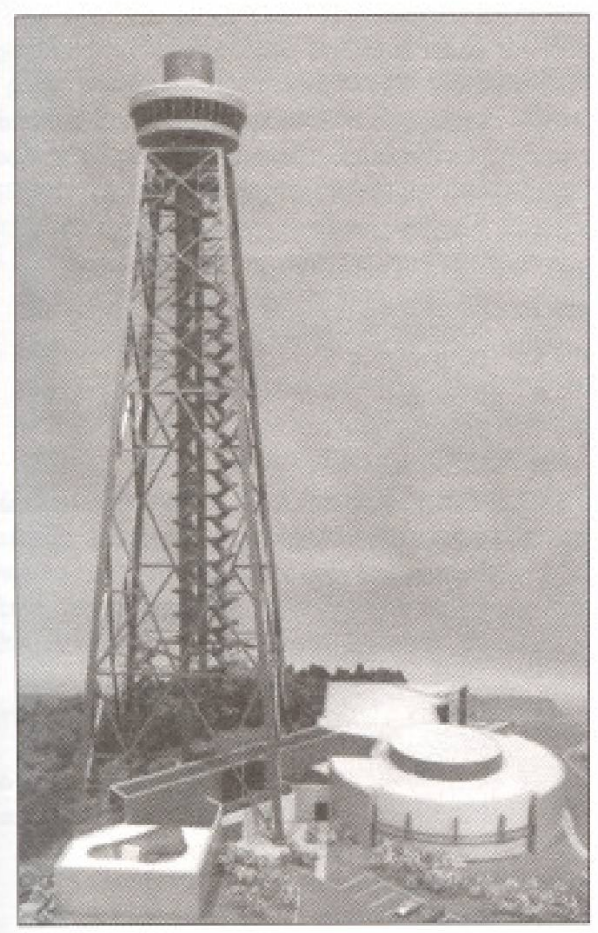

La tow ed le Cenire des sciences.
Ces quatre zones sont : le Quartier des contremaîtres, les Anciennes centrales, la centrale Shawinigan 2 et la Rotonde. La zone des Anciennes centrales concentre l'essentiel des installations muséographiques du secteur Hydro-Québec. La Centrale N.A.C, désalfectée depuis le milieu des annees quarinte, est entièrement recyclés à des lins de mussographie. C'est dins cette zone que l'on trouve aussi les vestiges de l'ancienne centrale Alcan 16 qui sont mis en valeur dans le cadre du projet. Le reste de cette zone comprend des aménagements de plein air et des services de restauration, notamment une vaste termasse qui surplombe les westiges de la Centrale Alcan $16 . \hat{A}$ proximité de cette zone, il y a la Centrale Shawinigan 2 qui fonctionne toujours avec ses équipements datant du début du siècle. Cette centrale, elassé paimi les vingt-cing plus grandes réalisations du gếnie canndien au cours des cent denières années, pourm être visitée en compagnie de guides. Entin, à la Rotonde, où se trouve le centre d'archives et les espaces gui seront mis â lat disposition des hénévoles du Parc, une exposition est présentée dans l'ancien poste de conduite du résenu de la Shawinigan Water and Power:

\section{LA CITÉ DE L'ÉNERGIE : UN LIEU MUSÉoloGIQUE MULTI- THÉMATIQUE UNIQUE AU QUÉBEC}

Au moment où la phase de programmation a été lancée, à la fin de l'ćce 1994, un solide travail prépanatoire avait dejà été réalisé par' les experts d'Hydio-Québec, par l'équipe du Centre d'interprétation de I'indusirie et pat plusieur chercheurs universitaires, La phase de progranmátion a permis entre autres de produire une thématique préliminalire ainsi que les programmes de muséographie, de consiruction et d'exploitation de l'institution. Au printemps 1995, la pluse de réslisation proprement dite étail lancết.

Contrairement a la plupart des grands projets muséologriques, la Cité de l'énergie est implanté sur un site qui fait intrinscquement partie du discours ha mettre en valeur et dont l'exploation constitue en soi une expénence très riche. Il est donc exsentiel d'ellaborer l'ensemble de l'expérience museologique en tenant comple de cos " higures imposés " et en les inscrant de faģon optimale dans le discours global de l'institu- tion. Outre le site physique, ces éléments déjầ présents sont constitués d'un bấtiment de centrale hydroélectrique construit en 1900, des vestiges d'une autre centrale légèrement plus jeune, d'une centrale construite en 1911 et toujours en exploitation ainsi que d'une ancienne salle de contrôle đéjà réaménagée en centre d'interprétation. On envisgeait finalement d'ajouter une tour d'observalion de 100 mètres afin de faciliter l'observation du site et de constituer un $c l e$ ment signal fort. En élaborant la thématique globale de l'institution, on s'est done appliqué a conférer a chacun de ces eléments du parcours une vocation thématique qui corresponde à sa nature et qui contribue à compléter l’expérience du visiteur.

Pour ce qui est de la thématique globale, elle consiste, de façon schématique, à utiliser le siecle d'histoire de Shawinigan comme enveloppe pour véhiculer plusieurs sousthematiques qui s'y fondent de façon naturelle. Parni ces autres thémaliques on trouve la vulgarisation des principes scientifiques qui sont a la base des grandes industries locales ainsi que des technologies industrielles qu'on y utilise et des produits qui en résultent. Certains éléments liés à l'histoire sociale et geographique de la région sont également mis en valeur. Même si l'histoire sert de fil conducteur au developpement thématique proposé, la thématique dominante est réellement du type "science-technique - societe "(STS) avec une forte emphise technologique. Ia mise en valeur de cette thématique à l'intérieur d'une logique typiquement historique devrait cependant la rendre plus acoessible et intéressante aux differents publics vises par l'institution.

Compte tenu de la durée relativement importante de la visite propose (de trois a cing heures), il était essentiel de bien rythmer celle-ci en jouant sur la variété des expériences et des émotions offertes. C'est ainsi que la visite commence avec une inposante pressentation multimedia qui sert d'introduction a l'ensemble de la visite en présentant les points tournants de l'histoire de la ville à travers les personnages et les situations qui ont marqué cette histoire. La particularité de cette présentation tient d'abord au fait qu'elle a lieu dans une salle circulaire dont l'estrade qui supporte les sièges effectue une rotation complete au fur et à mesure que l'histoire se déroule en remontant dans le temps. La salle d'exposition per- 
manente, qui se déploie en couronne autour de la salle multimédia, sert alors d'arrière-scène à la présentation, ses grands eléments muséographiques ctant mis en valeur au moment opportun.

Cette concordance des époques entre la sille multimédia et la salle d'exposition permanente permet un aller-retour dans le temps puisque les visiteurs sont $d^{t}$ abord emportés du present vers ce passé avant qu'on ne les invite a debarquer en quelque sorte dans $c e$ passé, lors du dernier tableau du multimédia, pour reparcourir le siècle en explorant l'exposition permanente qui suit également une trame chronologique qui va, cette fois-ci, d'hier à aujourd'hui. Alors que le multimédia privilégie d'abord la dimension humaine de l'aventure Shawinigan, l'exposition permanente se concentre sur ses aspects scientifiques et technologiques, tout en onservant évidemment sa trame historique.

En plus de suivre une progression chronologique, la visite de l'exposition permanente progresse au fil de quatre zones qui correspondent en même temps à des ćpoques, à des lieux et à des thématiques trè̀s spécifiques. Cette spécificité se transpose dans des environnements et des moyens muséographiques qui s'inspirent des lieux et des eproques en cause et qui sont done ainsi eux-mêmes porteurs d'information. Cette évolution des ambiances et des moyens contribue en outre à rythmer l'expérience du visiteur qui n'a plus l'impression de visiter un musée ou un centre d'interprétation mais s'imagine plutốt qu'il s'est évadé daus quelque repli de l'espace-temps historique de Shawinigan.

\section{UN SITE À EXPLORER}

Au sortir de l'exposition permanente, le visiteur possède l'essentiel des message qu' on a voulu lui transmettre. Il est prêt ă découvrir le site et ses vestiges. Un ascenseur l'emporte alors vers l'observatoire juché au sommet d'une tour de 100 m. $A$ cette hauteur, il contemple un panorama exceptionnel et découvre, à l'aide de supports muséographiques appropriés, la géographie du lieu; géographie physique dans l'axe nord-sud, géographie humaine dans l'axe est-ouest.

Le visiteur emprunte un petit bac pour atteindre la zone des vieilles centrales qui constitue la seconde partie de sa visile. II pénètre d'abord dans le sous-sol de la vieille centrale désaffectée de la Northern Aluminum Company qui date de 1900. Une reconstitution des conduites forcées et de certains éléments de machines lui permet de comprendre le fonctionnement de la centrale. Au rez-de-chaussée du bâtiment, il découvre la collection de patrimoine industriel de la Cité de l'énergie. Ces pièces proviennent essentiellement des premières installations hydroélectriques et indusurielles de la région et sont mises en valeur avec des moyens muséographiques originaux qui permettent d'en comprendre le fonctionnement. Puis, le visiteur se rend at une aire de repos aménagée sur les vestiges d'une autre centrale, avant de visiter lá centrale Shawinigan 2. Construite en 1911, la centrale utilise toujours les mêmes machines hydroćlectriques qui étaient à l'époque les plus puissantes du monde.

La visite se ternnine daus l'ancien édifice de la Rotonde où un specticle vidé́, sons et lumières amène le visiteur à généraliser les propos véhiculés lons de la visite. On y fạit entre autres le point sur l'évolution des anciennes cités industrielles dans le contexte de mondialisation de l'économie et de restructuration du tissu industriel mondial.

Les différentes étapes de la visite de la Cité de l'énergie ont ếté conçues de façon à produire un effet cumulatif dans l'esprit du visiteur et à lui transmettre l'ensemble des messages prévus à l'élape de progranmation. En variant les types d'expériences et en s'efforçant toujour's d'accoler très naturellement des contenus de communication pertinents à chacun des lieux visites, on a élahoré, à la Cité de l'Énergie, un circuit de visite qui sera à la fois une experrience enrichissante et divertissante pour un très large public.

$$
\text { -.. }
$$

La Cité de l'Énergie accueillera ses premiers visiteurs en juin 1997. C"est à partir de cette date qu'elle aura à relever son principal défi : placer le Centre-de-lá-Mauricie sur la carte des grandes destinations touristiques du Quéthec et contribuer à l'expansion de l'industrie touristique régionale.

\section{NOTES ET RÉFÉRENCES}

1 "Shawinigan, première ville epensects au Canada". Le Devoir. le 8 juillet $1996, \rho_{\text {. Al }}$ et A8.

2 Itidem.

3. Ses origines sont encore plus lointaines, En effet. des 1947. la Shawinigan Water and

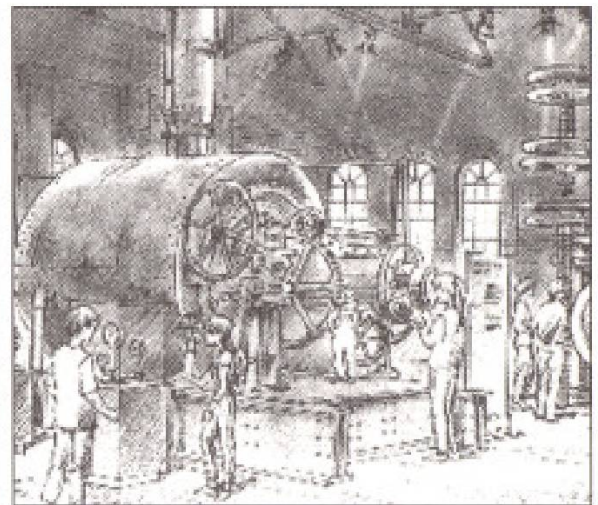

Esquisse NAC.

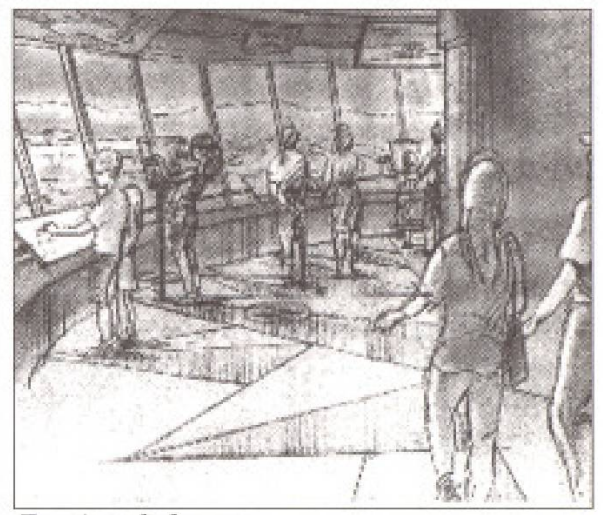

Esquisse de la tour.

Power Company, qui s'apprête à feter son cinquantième anniversaire l'année suivante, projette de erócer un musće de l'électricité à Shawinigan.

4 Corporation du Centre d'interprétation de l'industrie de Shawinigan inc., (1995) Prograwne de mise en oeuve, p. 1-5. Il y aurait toutefois lieu de nuancer ledit isolement. Shawinigan n'est certes pas situee à proximité immédiate d'un grand centre de population. Directement reliée par autoroute à Montrétal ef à Québec, Shawinigan jouit au contraire d'une position plus centrale qu'excentrique, surtout si on la compare au Lac-Saint-Jean, a Sudbury, à Curaquet.

5 Ibiden.

6 Ibidem.

7 Idem, p. 5-1.

8 Idem, Annexe C, p. 1.

9 COOPERS L LYRAND (en association avec les Ingénieurs-Conseils Pierre Larocque \& Associés), Le Centre d'interpétation de I'industrie de Shawinigan - Erude de viabilite, rappoit final, Montréal, aodit 1991 .

10 Janody Marketing inc., (1996) Orientations strategiques de marketing, tableau 27.

11 Corporation du Centre d'interpuétation de 1 'industrie de Shawinigan inc., Programme de mise en ocuvre, p. 1-6.

12 Ibidem.

13 Ibiden.

14 Entretien du 10 juillet 1996. 